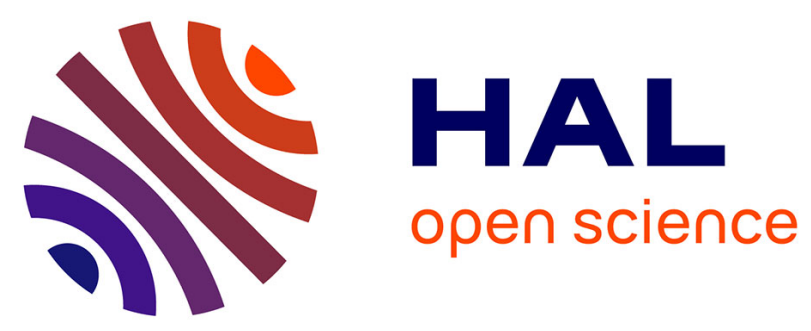

\title{
Kalapani : le trauma de la traversée dans la migration des demandeurs d'asile tamouls du Sri Lanka
}

Marie-Caroline Saglio-Yatzimirsky

\section{To cite this version:}

Marie-Caroline Saglio-Yatzimirsky. Kalapani : le trauma de la traversée dans la migration des demandeurs d'asile tamouls du Sri Lanka. Migrations Société, 2015. hal-01279243

HAL Id: hal-01279243

https://hal-inalco.archives-ouvertes.fr/hal-01279243

Submitted on 1 Mar 2016

HAL is a multi-disciplinary open access archive for the deposit and dissemination of scientific research documents, whether they are published or not. The documents may come from teaching and research institutions in France or abroad, or from public or private research centers.
L'archive ouverte pluridisciplinaire HAL, est destinée au dépôt et à la diffusion de documents scientifiques de niveau recherche, publiés ou non, émanant des établissements d'enseignement et de recherche français ou étrangers, des laboratoires publics ou privés.

\section{(1)(1) $\$(0)$}

Distributed under a Creative Commons Attribution - NonCommercial - ShareAlikel 4.0 


\title{
Kalapani : le trauma de la traversée dans la migration des demandeurs d'asile tamouls
}

\author{
du Sri Lanka
}

Marie-Caroline Saglio-Yatzimirsky, CESSMA, Inalco, Sorbonne Paris Cité, IRD Université Paris Diderot, Université Paris Diderot Case 701775205 Paris Cedex 13

\section{Résumé}

Si les recherches cliniques en psycho-traumatologie et psychanalyse transculturelle auprès de migrants réfugiés ont largement abordé l'avant et l'après de la migration, rares sont les études s'intéressant à la traversée elle-même. La métaphore hindoue de la traversée des eaux noires (kalapani) rappelle que quitter son pays peut constituer une transgression. A partir de deux cas cliniques de patients réfugiés tamouls du Sri Lanka, nous développerons notre hypothèse initiale de la traversée de l'océan comme espace-temps du trauma. On repère deux constellations dans l'expression des patients: soit le voyage est irreprésentable car vécu comme mort symbolique, soit il envahit la pensée sous forme de profusion d'images traumatiques. Nous verrons dans un second temps comment le travail d'élaboration dans la consultation de psycho traumatologie transculturelle permet de penser la traversée comme voyage et lien plutôt que fuite. C'est en l'occurrence les liens de caste qui se réactualisent, ceux-là mêmes qui avaient été menacés par le kalapani. Par son cadre et sa dynamique, la consultation peut constituer un port d'accostage pour des patients « désorientés ».

\begin{abstract}
If clinical researches in psycho traumatology and transcultural psychoanalysis with migrants and refugees have largely addressed the before and after of migration, few studies have dealt with the crossing itself. Hindu metaphor for crossing the black water (kalapani) recalls that leaving the country may be a transgression. Based on two clinical cases of asylum seekers, we will develop our initial hypothesis that the crossing of the ocean is the space-time of the trauma. Two suffering expressions are analyzed: either the journey is unrepresentable because it is lived as a symbolical death, either the journey invades the patient's psyche with a profusion of traumatic images. We will then examine how the work developed in the psycho trauma consultation suggests the crossing as a travel and a link instead of a leak. Caste ties may be actualized, even those who had been threatened by the kalapani. Through its setting and its dynamics, the consultation may be a docking port for "disoriented" patients.
\end{abstract}

Mots-clés: migration, demandeurs d'asile, traversée, psycho trauma, clinique, transculturel, kalapani, tamoul 
« Mais n'as-tu pas peur, dit-elle, de perdre ta caste ? De traverser l'Eau noire et de te retrouver sur un bateau avec toutes sortes de gens ? » A. Ghosh, Un Océan de Pavots.

À écouter Karan parler de sa fuite, à entendre la désorientation de Sandhya depuis son arrivée en France, la traversée des mers est funeste. Tous deux sont tamouls du Sri Lanka, et comme certains de leurs compatriotes ont dû fuir la guerre dans des conditions dramatiques. Ils sont aujourd'hui demandeurs d'asile en France et ont été, l'un amené par un ami, l'autre orientée par un foyer d'accueil dans la consultation de psycho-traumatologie du service de psychiatrie de l'hôpital Avicenne (93).

Après plusieurs mois de travail psychothérapeutique ${ }^{1}$, notre thèse clinique est que la traversée est au cœur du trauma du réfugié ${ }^{2}$. Si les recherches en psychopathologie et psychanalyse transculturelle ont largement abordé l'avant et l'après de la migration, le premier pensé comme moment de préparation nécessaire par des rituels collectifs ${ }^{3}$, le second présenté comme un bouleversement des repères culturels ${ }^{4}$, rares sont les études s'intéressant au passage lui-même, lorsque le trauma est dans le fait même d'avoir traversé ${ }^{5}$. Parmi les raisons possibles de cette occultation est la spécificité de l'investissement psychique de l'espace-temps de la traversée. Entre le moment du départ et le moment de l'arrivée, est un abîme d'angoisse qui interdit toute représentation ou qui, à l'inverse, est chargé d'images sidérantes ${ }^{6}$. Dans la traversée, le lien semble se briser entre ici et là-bas, et c'est là que réside la clé du clivage. A partir des cas cliniques de Sandhya et $\mathrm{Karan}^{7}$, nous développerons notre hypothèse initiale de la traversée comme espace-temps du trauma prenant forme soit de voyage irreprésentable car vécu comme mort symbolique, soit envahissant la pensée sous forme de profusion d'images traumatiques.

Cette approche clinique est articulée à une perspective anthropologique. Pour mieux appréhender l'angoisse psychique des demandeurs d'asile tamouls, on se réfère au schéma culturel de kalapani (lit. eaux noires), le tabou de l'interdiction pour les Hindous de haute caste de traverser l'océan. Dans la tradition des siècles derniers, les castes supérieures $(d v i j a)^{8}$ sont tenues de vivre sur la terre mère où peut s'accomplir le dharma. ${ }^{9}$ Or en traversant les

\footnotetext{
${ }^{1}$ Ces consultations se sont tenues entre novembre 2010 et mars 2013 dans le service de psychiatrie d'Avicenne, Bobigny où j'exerce comme psychologue clinicienne consultante externe.

${ }^{2}$ On distinguera à l'instar de T. Bokanowski le traumatisme «figurable», le traumatique ou « défaut de pareexcitant» et le trauma, c'est-à-dire l'action du traumatisme sur l'appareil psychique. V. BOKANOWSKI, Thierry, "Traumatisme, traumatique, trauma“, Revue française de psychanalyse, (3) vol. 66, 2002.

${ }^{3}$ ROUSSEAU, Cécile; SAID, M. Taher, GAGNE, Marie-J., BIBEAU, Gilles (1998), Between Myth and Madness, Dreaming the Departure. Premigratory Period for Young Somali Refugee, in Culture, Medicine and Psychiatry, (22): 385-411.

${ }^{4}$ MORO, Marie-Rose, Psychothérapie transculturelle des enfants de migrants, Dunod, Paris, 1998.

${ }^{5}$ En revanche, les études d'anthropologie et de géographie ne manquent pas qui étudient précisément les trajectoires des migrants et qui abordent les conséquences physiques et mentales de ces migrations.

${ }^{6}$ LACHAL, Christian ; OUSS-RYNGAERT, Lisa ; MORO Marie-Rose, Comprendre et soigner le trauma en situation humanitaire, Dunod, Paris, 2003.

${ }^{7}$ Les noms des patients ainsi que certains détails biographiques ont été volontairement modifiés par souci de confidentialité.

${ }^{8}$ La caste au sens de jati (espèce, groupe de naissance) est l'institution fondamentale qui organise le monde socio-religieux hindou. Ce terme vernaculaire pour désigner le groupe endogame d'appartenance oriente les représentations et partie des pratiques sociales et professionnelles selon les critères de pureté et de pollution. Réalité locale, multiforme et changeante, la caste n'en reste pas moins prégnante dans l'organisation de la vie sociale en Inde aujourd'hui.

${ }^{9}$ L'histoire contemporaine de Sri Lanka s'est forgée dans une relation ambiguë par rapport à leur puissant voisin, L'Inde. L'étude de Jonathan SPENCER (2014) montre comment la situation insulaire a favorisé les
} 
eaux, il n'est plus possible de maintenir les lois de caste qui garantissent le statut, c'est-à-dire les règles de commensalité et de mariage. C'est la traversée elle-même qui est transgressive: là, le risque de se mêler à des étrangers ${ }^{10}$ et à plus impur que soi et de s'alimenter d'une nourriture préparée par des castes inférieures provoque la déchéance. La traversée figure alors la transgression d'un interdit et le déclassement.

Pourquoi convoquer le motif métaphorique de kalapani à l'écoute des patients tamouls hindous réfugiés dans une consultation de psychotraumatologie? Ce faisant on pose la question d'un possible écho psychique d'une telle croyance ${ }^{11}$. Ce qui nous intéresse ici est l'émergence de la dimension culturelle autour de la transgression des règles propres à la communauté. Il y a certes bien d'autres modalités pour les migrants de vivre le voyage (Rousseau et alii, 1998). Toutefois la potentielle richesse du voyage est occultée dans la migration subie et forcée: si le voyage mène à une nécessaire reconfiguration identitaire, c'est d'abord sous le seau de la perte et de la transgression.

Notre perspective à la fois clinique et anthropologique se précisera dans la dernière partie de notre propos : nous verrons en effet comment la consultation thérapeutique permet d'effectuer un travail de «liens». Celui-ci va faire émerger une institution sociale fondamentale : celle de la caste. De même que la protection du statut de caste est au centre du tabou de kalapani, c'est dans la praxis de la caste, autrement dit lorsqu'il s'agit de parler mariage et repas, que la situation de caste prend tout son sens. C'est par elle que peuvent se reformuler les liens sociaux et se réimposer le symbolique.

\section{Un dispositif clinique: la prise en charge psycho thérapeutique des migrants tamouls}

La consultation de psycho traumatologie du service de psychiatrie de l'hôpital Avicenne constitue un des lieux de prise en charge psychiatrique et psychologique des migrants demandeurs d'asile. Souvent orientés par les réseaux sociaux du Département, ces patients sont arrivés en France récemment et en général seuls, après des parcours d'une extrême violence physique et psychique. Le «trauma intentionnel ${ }^{12}$ se double d'une expérience potentiellement traumatique, celle de la migration elle-même, qui ajoute à l'expérience traumatique initiale (mort de proches en situation de guerre, torture, viol, etc.) la perte à la fois réelle et symbolique de l'espace culturel d'origine. Dans la consultation trauma sont accueillies des personnes présentant un syndrome post-traumatique au sens du DSM-V (reviviscences, dissociations, dépression, etc) ${ }^{13}$. Elles sont en général orientées vers des prises en charge bifocales, à la fois psychiatrique et psychothérapeutique, cette dernière pouvant être aménagée dans un cadre individuel ou groupal.

\footnotetext{
connexions et migrations tandis que la situation coloniale positionne différemment Sri Lanka et l'Inde par rapport à l'Empire britannique. Pour ces raisons, la société sri lankaise, en particulier hindoue, propose une représentation spécifique et distincte de celle de l'Inde de la société tamoule locale et des liens de caste.

${ }^{10}$ Les mlecchas, terme issu du sanskrit, désignent dans l'Inde ancienne les étrangers qui ne suivent pas les préceptes de l'hindouisme et donc les règles de caste.

${ }^{11}$ Cette croyance est certes ancienne, mais des études récentes montrent qu'elle n'a pas entièrement disparue de la société hindoue. V. l'étude de TROUILLET, Pierre-Yves, "Parcours et expériences de brahmanes indiens officiant dans les temples tamouls de l'île Maurice", Communication, Colloque "Les Indiens et la mer", INALCO, 5 juin 2013. V. également SERVAN-SCHREIBER, Catherine « La traversée des Eaux-noires (kalapani) à Maurice et dans la Caraïbe. Histoire, usages et perceptions de l'hindouisme moderne » communication, 6 mai 2011, EHESS, séminaire Kalapani (CEIAS).

12 SIRONI, Françoise, Bourreaux et victimes. Psychologie de la torture, Odile Jacob, Paris, 1999.

13 Le Manuel diagnostique et statistique des troubles mentaux (Diagnostic and Statistical Manual of Mental Disorders- DSM) est un ouvrage de référence publié par la Société américaine de psychiatrie (APA) classifiant et catégorisant des critères diagnostiques et des recherches statistiques de troubles mentaux spécifiques. La cinquième version du DSM est parue en 2013.
} 
Les patients dont il est question ont vécu de manière fort différente le passage de là-bas à ici. La France est vécue comme terre d'accueil ou terre d'exil, quelque fois valorisée, souvent destination inconnue qu'il n'a pas été possible d'élaborer. La consultation de psychotraumatologie intègre la dimension politique et sociale de cette souffrance tout en s'adressant à des patients isolés dans leur trauma. L'un des objets du travail thérapeutique va être de pouvoir élaborer le voyage vécu comme la perte issue d'une "migration forcée » en un déplacement qui peut être positivement élaboré par la réélaboration et le tissage de liens psychiques et sociaux.

Il n'est pas question de reprendre dans le cadre de cet article l'ensemble de l'histoire des migrations politiques des tamouls du Sri Lanka en France ${ }^{14}$. Rappelons toutefois que d'une part, la fin officielle de trente ans de conflit n'a pas limité les départs et les demandes d'asile en France ${ }^{15}$. D'autre part, les populations qui arrivent en France sont divisées, ce qui réduit les possibilités d'entraide ou de mobilisation collective ${ }^{16}$ entre compatriotes ${ }^{17}$. Notre étude s'intéresse aux réfugiés actuels du conflit tamoul-cinghalais, qui comprend une majorité de castes intermédiaires, arrivés dans une troisième vague de migration en France, après les premiers venus dans les années soixante-dix, plutôt issus des castes supérieures, puis une seconde vague dans les années quatre-vingt-dix qui compte majoritairement des castes basses et intermédiaires et plus directement engagées dans le conflit. Les oppositions de caste et de statut redoublent les prises de positions politiques. Les derniers arrivés sont aussi les moins bien perçus sur un territoire francilien qui est lui-même en proie à des oppositions entre factions rivales.

Le contexte politique du migrant, sa «carrière » de demandeur d'asile, et l'espace temps "en suspension (...) fait d'attente, de précarité et d'insécurité administrative ${ }^{18}$ avive son sentiment d'illégitimité qui n'est pas sans rappeler la « double absence » de A. Sayad ${ }^{19}$ : il est à la fois délégitimé au Sri Lanka car il en est parti et illégitime en France de par son statut de migrant, éventuellement de sans papiers. Rappelons que depuis 2009, lorsque l'armée sri lankaise écrase la résistance des combattants du mouvement des Tigres de Libération de l'Eelam Tamoul (LTTE) et officialise la fin du conflit, le Sri Lanka sort de la liste de l'Office Français de Protection des Réfugiés et Apatrides (OFPRA) des pays considérés comme dangereux. C'est une non reconnaissance pour les réfugiés tamouls du Sri Lanka qui a pour conséquence de minimiser leur possibilité de légalisation en France et d'accroître les possibilités d'un retour au pays qui signifie la mort pour certains patients ${ }^{20}$. La

\footnotetext{
${ }^{14}$ Nous renvoyons aux travaux de GOREAU-PONCEAUD, Anthony, "Routes et antiroutes de l'immigration tamoule sri-lankaise : des camps du Tamil Nadu à la Chapelle ", http://www.mshs.univ-poitiers.fr/migrinter/emigrinter/201208/e-migrinter2012_08_026.pdf e-migrinter, n8, 2012 et GOREAU-PONCEAUD, Anthony, "L’immigration sri lankaise en France - Trajectoires, contours et perspectives", Revue Hommes et Migrations, $\mathrm{n}^{\circ} 1291$, mai-juin 2011.

${ }^{15}$ Comme le rappelle A Goreau-Ponceaud «l'OFPRA (a) même enregistré en 2009 une hausse des premières demandes d'asile sri-lankaise (+33\% par rapport à 2008)» (2012 : 28). V. également les chiffres du Rapport de la mission de l'OFPRA en République démocratique et socialiste de Sri Lanka du 13 au 27 mars 2011. http://www.ofpra.gouv.fr/documents/misSLK_2011.pdf

${ }^{16}$ V. MANTOVAN, Giacomo, "Les récits de vie des demandeurs d'asile tamouls - Vers une mémoire collective ? "Revue Hommes et migrations, $\mathrm{n}^{\circ}$ 1291, mai-juin 2011

${ }^{17}$ La société tamoule sri-lankaise peut être divisée globalement en deux communautés, les Tamouls dits sri lankais installés avant la colonisation britannique, considérés comme «autochtones » et qui en tirent une certaine supériorité, et les Tamouls dits indiens « des plantations », souvent issus de basse caste du Sud de l'Inde, arrivés au XIXe siècle comme main-d'œuvre embauchée par les Britanniques pour travailler dans les plantations du centre de Ceylan.

${ }^{18}$ IRAGO, Daniel, "Le demandeur d'asile aux prises avec le dehors et le dedans", Cliniques, Eres, 2011/2 - № 2 , 2011.

${ }^{19}$ SAYAD, Abdelmalek, La double absence. Des illusions de l'émigré aux souffrances de l'immigré, Seuil, 1999.

${ }^{20}$ La mission de l'OFPRA (2011) constate la pacification de la zone Nord du Sri Lanka « en voie de reconstruction».
} 
consultation de psycho traumatologie s'élabore ainsi dans un contexte institutionnel et politique global et propose un cadre suffisamment élaboré pour assurer un soutien psychothérapeutique.

\section{Trauma de la traversée: kalapani, transgression et perte}

\section{Sandhya : la traversée comme perte inélaborable ${ }^{21}$}

Sandhya est une femme tamoule du Sri Lanka de 45 ans, de caste supérieure, placée dans un foyer d'accueil pour demandeurs d'asile avec ses trois fils majeurs. Sandhya a perdu son mari il y a vingt ans, tué par balle sur le seuil de sa maison, alors qu'il venait d'être appelé au dehors, interrompu au milieu d'un repas familial. La famille de Sandhya étant continuellement menacée, une partie a fui en Suède. Deux de ses frères restés au Sri Lanka ont été tués, de même qu'un cousin et que son beau-frère. En 2008, ayant réussi à économiser assez d'argent, elle fuit le Sri Lanka avec ses fils pour la France.

Madame se présente dans le service d'Avicenne alors que sa demande d'asile à l'OFPRA vient d'être rejetée. Elle est prise en charge par un psychiatre qui diagnostique une dépression sévère. Par ailleurs, le contexte d'arrivée en France semble favoriser l'expression de très fortes angoisses de séparation. En effet, la progressive prise d'autonomie de ses fils qui cherchent un emploi et vivent leurs premières aventures amoureuses est source d'angoisse sinon de désorganisation pour Sandhya, qui a élevé ses enfants de manière symbiotique. Après quelques mois de prise en charge psychiatrique, elle est orientée vers le "groupe trauma », pour d'une part tenter de faire bouger un trauma dit « enkysté », pour d'autre part l'aider à élaborer les séparations qui se profilent. Elle est prise en charge mensuellement par ce groupe thérapeutique qui réunit plusieurs soignants (psychiatres, psychologues, infirmiers) avec le patient. Un élément important de cette consultation est la place de l'interprète, en l'occurrence tamoul d'Inde : son positionnement est rendu complexe dans le cas des Tamouls du Sri Lanka en raison de sa plus ou moins grande empathie et bienveillance à l'égard de leurs homologues de Sri Lanka ${ }^{22}$.

Dans les paroles de Sandhya, la question de désorientation, de la déterritorialisation, du déclassement sont des motifs récurrents qui prennent leur source dans l'abîme d'angoisse de la traversée. Pourtant, paradoxalement, de la traversée, Sandhya ne dit quasiment rien. Le voyage proprement dit est à peine évoqué et a les contours de ces traversées erratiques avec des escales improbables, tributaires des opportunités offertes par les passeurs: un premier vol emmène la famille de Jaffna à Singapour, un second fait le vol Singapour-Paris. Pendant tout ce temps, Sandhya vit l'étrangeté, à la fois forcée et consentie, dans une ambivalence difficilement supportable, et qui se solde en clivage.

Une fois en France, ses difficultés psychologiques s'aggravent: séparation et avenir incertain de ses fils, solitude loin du pays natal la plongent dans une grave dépression. L'arrivée dans un pays dont elle ne parle pas la langue - et Sandhya ne souhaite pas

\footnotetext{
21 En psychanalyse, l'élaboration désigne le travail psychique de symbolisation, qui permet par la parole de maîtriser l'excitation, et dans le cas du trauma, de sortir de la répétition mortifère. Le trauma est dit inélaborable quand il n'y a pas de processus de symbolisation ou de secondarisation possible qui permettrait de sortir de l'effraction psychique et de la dépasser.

22 Les Tamouls du Sri Lanka et du Tamil Nadu, Etat du Sud de l'Inde, n'ont ni la même histoire, ni la même représentation de leur communauté, ni même une langue identique. En l'occurrence, les Tamouls du Tamil Nadu appartiennent à ce voisin puissant, l'Inde (v. note 10), qui a pris un ensemble de décisions politiques cruciales pour les Tamouls du Sri Lanka tout au long de la guerre. Une forme de condescendance (au sens d'habitus de Pierre BOURDIEU) peut ainsi se faire ressentir chez certains interprètes tamouls d'Inde à l'égard des Tamouls srilankais.
} 
l'apprendre - est aggravée par son impression de déclassement: elle et ses fils «sont entassés » dans une seule pièce, eux qui avaient leur maison au Sri Lanka; les relations de service entre compatriotes sont monétarisées contrairement à celles entre voisin au pays; personne n'a de considération pour elle pourtant de caste supérieure. Ainsi Sandhya souffre de se sentir prise dans plusieurs espaces de références qui semblent irréconciliables et que ne partagent pas ses fils. Le clivage est radical entre deux mondes, son monde d'origine où elle a laissé ses références, sa maison et son mari, et le monde d'ici, dans lequel ses fils ont un pied bien engagé.

D'autant qu'un reproche lui est adressé par sa famille. Sandhya a 20 ans quand elle devient veuve, ce qui suppose dans sa vie de femme hindoue endosser un statut difficile. Au pays, sa soudaine position de veuve ${ }^{23}$, lui a été reprochée par sa belle-sœur, celle dont le mari - frère de Sandhya - a été tué. La menace est ainsi endogène, Sandhya pense avoir transgressé un certain nombre de règles propre à sa famille et à sa caste.

Cette menace endogène est redoublée par la menace exogène et propre au pays d'accueil : celui-ci est sans loi $^{24}$. Il vient menacer ses principes tels que maintenir la mémoire $\mathrm{du}$ père, garder ses fils auprès d'elle, arranger leur mariage. Prise dans des injonctions contradictoires, devoir de fuir le monde traditionnel, impossibilité d'accepter les règles du pays d'accueil, elle refuse les interactions avec celui-ci, pour elle et pour ses fils. Même majeurs et occupés à chercher un emploi, elle exige que ces derniers reviennent déjeuner à la maison de la nourriture préparée par ses soins.

Le parcours administratif de Sandhya pour obtenir l'asile politique suppose psychiquement la séparation d'avec le Sri Lanka et d'avec son mari mort : elle est impossible à élaborer. C'est donc un sentiment de "déterritorialisation» qui la plonge dans les eaux noires de la dépression, déterritorialisation au sens deleuzien où elle a quitté le territoire existentiel qui circonscrit le champ du familier, marque les distances avec l'étranger et protège du chaos ${ }^{25}$. Rapporté au monde socioreligieux hindou, cette déterritorialisation se traduit par la désorientation et le déclassement de la caste.

\section{Karan: la traversée comme travestissement}

Karan jeune journaliste, aîné d'une famille de caste supérieure de trois enfants, soupçonné d'intelligence avec le LTTE par les autorités sri lankaises, a été emprisonné et torturé. Par ses connexions il a pu soudoyer les gardes pour sa sortie. Puis un ami l'aide à s'enfuir par avion : il doit pour cela se «déguiser en riche ", "se raser le crane, mettre des lunettes de soleil et ne prendre avec lui qu'une valise légère comme s'il voyageait en touriste ». Il prend alors l'avion pour un trajet qui lui paraît «insensé » : au gré des contacts, il débarque en Afrique de l'Ouest, où il passe une semaine, puis reprend un vol pour Hong Kong, enfin de là un vol pour la France. Son périple dure plusieurs mois, et en traversant ces milliers de kilomètres, il sent se rompre les amarres qui le lient à son pays. Karan n'a pas pu prévenir ses parents de son départ et ne les a pas revu depuis son emprisonnement.

Nous recevons Karan en consultation individuelle tous les quinze jours. Karan n'a pas

\footnotetext{
${ }^{23}$ Sur l'ambivalence de la figure féminine et en particulier sur la culpabilité des veuves, V. KAKAR, Sudhir, The Inner World, A Psycho-Analytic Study of Childhood and Society in India, Oxford Universiy Press, 1982.

${ }^{24}$ On entend le terme de loi à la fois au sens socio-anthropologique de normes sociales qui ordonnent les actions, et au sens symbolique de la loi telle qu'on la retrouve chez Freud : règles organisant les rapports de sexe et de génération dans un société. Enfin, dans le cas présent, la loi est aussi celle du surmoi psychanalytique qui structure moralement de psychisme du sujet.

${ }^{25}$ DELEUZE, Michel ; GUATTARI, Felix, Capitalisme et schizophrénie, t. 2 : Mille-plateaux, Minuit, Paris, 1980.
} 
souhaité d'interprète, et la consultation se fait en anglais, qui n'est ni la langue maternelle du patient ni la nôtre. Lors de son premier entretien, il dit ne pas pouvoir dormir, être envahi par des images de violence, avoir des pertes de mémoire. La souffrance s'exprime somatiquement par des douleurs dans tout le corps - un corps qu'il "ne reconnaît plus » tant il a "changé » sous la torture- et des maux de tête intenses. Son discours est confus et charrie la culpabilité du survivant qui a abandonné aux griffes de l'ennemi sa famille et ses amis de lutte. Le trauma est bien dans la rupture qu'occasionne la traversée, rupture qui est aussi un travestissement de Karan, qui s'énerve d'avoir perdu ses moyens de lutte, de ne pas pouvoir

témoigner. Repenser à la traversée le plonge dans un questionnement douloureux sur cette migration forcée qui l'a fait devenir " un autre », étranger à lui-même, à son propre corps, à son métier.

Ce qui frappe dans l'histoire de Karan est son extrême solitude psychique, alors qu'il est inséré dans un réseau de compatriotes. Karan a pourtant l'atout de la langue, lui qui maîtrise le cinghalais, le tamoul et l'anglais. Ayant rejoint une cérémonie à la mémoire des jeunes combattants tamouls du Sri Lanka morts pour la cause, il raconte comment il se sent «dédoublé » : à la fois plein d'empathie pour de ces familles qui ont perdu un proche, à la fois dans l'impossibilité de partager leur peine. Impossible assimilation aux migrants car ce serait entériner la rupture d'avec le pays et la perte d'une partie de son identité. Impossible rapprochement avec des familles en deuil car ce serait commencer à envisager la mort de son frère enlevé récemment au pays.

Quelques mois plus tard, à partir de la maison paternelle au pays, est organisé le mariage de sa sœur, qui doit permettre à la jeune fille de rejoindre ce nouvel époux qui vit à Londres. Ce mariage est vécu comme un déchirement par Karan, qui constate que sa mère a maintenant "perdu tous ses enfants », comme si la dispersion familiale ne pouvait être vécue que comme éclatement et perte et non comme sauvetage.

Il abandonne ses articles car il «n'entend que des mensonges ». Il se dit " déçu que les hommes ici ne poursuivent pas la lutte, oublient et se consacrent à leur petite vie reconstruite en France loin du pays ». Il a rompu toutes les appartenances : les liens familiaux, amicaux, et même professionnels. Karan a vécu son départ du pays comme une mort symbolique, comme la falsification de son identité. La traversée est le moment fondateur cette mort, lorsque le meurtre psychique a eu lieu. De fait il se pense mort, et lorsqu'il se rend à la convocation à la Cour Nationale du Droit d'Asile (CNDA), Karan exprime sa négativité dans son refus de répondre aux questions et de «coopérer» comme il lui sera reproché par les magistrats. Il dit dans la consultation à laquelle il apporte la lettre de refus de la Cour : "je souhaite que de ma mort au moins germe un arbre ».

\section{Sandhya : mariage et filiation}

La convocation à la CNDA suit d'environ un an le début de la prise en charge de Sandhya. Mieux préparée à l'entretien, elle obtient une réponse positive de la CNDA. En obtenant l'asile en France, Sandhya coupe symboliquement le lien avec sa mère " qui n'a pas su protéger ses fils» et avec le malheur que cette mère lui a légué. Cela émerge plus fortement chez Sandhya que la séparation d'avec son pays et son mari.

Toutefois l'angoisse de séparation et le clivage entre les deux cultures ressurgissent doublement autour de la question du mariage de ses fils. La nouvelle situation administrative a permis à ces derniers d'obtenir chacun un logement individuel, première difficulté pour Sandhya qui vit cet aménagement de manière contradictoire avec un modèle de référence hindou où les enfants partagent le toit familial jusqu'au mariage. Même vivant séparément, les fils reviennent encore déjeuner chez leur mère: élément fondamental qui cristallise la 
proximité de Sandhya avec ses fils, et élément de référence au système symbolique des castes et ses règles de pureté ${ }^{26}$. Surtout son fils cadet veut se marier, transgressant ainsi la traditionnelle hiérarchie des rôles dans la famille, qui d'une part choisi le partenaire, d'autre part privilégie l'aîné. La position de Sandhya et son intégrité sont brutalement menacées par les pratiques occidentales.

Plusieurs consultations vont être nécessaires pour que s'expriment les difficultés de Sandhya. A propos des velléités de mariage de son fils cadet, Sandhya commente : « il m'a oublié », se posant directement comme figure rivale de la fiancée. Elle va brandir initialement l'argument d'une probable incompatibilité de castes entre les fiancés, argument qui est à la fois une défense contre l'angoisse de la transgression des ordres d'alliance dans le monde hindou mais aussi contre l'angoisse de séparation. Sandhya se renseigne sur la jeune fille en faisant jouer ses réseaux de connaissance «là-bas». Il apparaît que les castes sont compatibles. Ce qui fait conflit se recentre alors sur la loi, celle du père et de la tradition, que Sandhya a charge d'appliquer. Elle ajoute : «Le petit ne connaît pas les castes; mais je suis pour un mariage qui va être strict au niveau de la culture (...) le cadet ne peut se marier avant l'aîné, sinon on croit que les aînés sont mauvais ». Ses angoisses ressurgissent d'autant plus fortement qu'elles lient culture et trauma : ses frères restés au pays, tous les deux tués, avaient fait des mariages d'amour. L'équation, probablement non consciente, est la suivante : si jamais mon fils cadet fait un mariage d'amour, il risque de lui arriver ce qui est arrivé à mes deux frères, qui avaient transgressé la loi symbolique.

Sandhya décide alors d'imposer la tradition dont elle se pense garante et entame les démarches pour marier l'aîné de ses fils : elle active ses réseaux familiaux et consulte l'astrologue au pays. L'aîné se montre pactisant et accepte la proposition qui lui est faite. Le mariage s'organise quelques mois plus tard avec une jeune fille de sa caste venue du Sri Lanka. Liant ainsi fête, ordre et séparation nécessaire, un thérapeute commente : "un mariage c'est une grande joie mais les parents pleurent aussi car c'est un moment où les enfants ne viennent plus manger à la maison ». Madame retrouve à travers les liens de caste respectés et le rituel (le repas et la fête) de quoi sortir de la transgression et se « reterritorialiser».

\section{Karan : territoire des liens de caste}

Les symptômes post-traumatiques de Karan s'aggravent dans les mois qui suivent le rejet de sa demande d'asile par la CNDA. En consultation, le travail consiste à réactiver les liens rompus par le trauma ou désinvestis. Il lui est proposé de réécrire son histoire, non pas comme il l'a fait pour les convocations administratives à l'OFPRA et à la CNDA, mais comme "témoignage ", afin de renouer avec son identité de journaliste qui est pour lui une manière de se situer personnellement par rapport à la guerre et de donner sens aux morts et disparitions de ses proches. Cette opportunité de mettre en mots certains éléments de son histoire, de la secondariser, de l'ordonner dans un espace-temps conciliable avec l'histoire dramatique de sa communauté, lui permet de sortir de l'évènementiel et de la singularité traumatique pour s'inscrire dans une Histoire collective. C'est au cours de ce travail que Karan peut alimenter son témoignage de sa propre histoire sans craindre le clivage du nonsens entre ici et là-bas. Sa famille n'est plus restreinte au seul noyau nucléaire qui se décompose mais s'élargit pour comprendre les ramifications européenne : alors qu'il prend contact avec sa sœur de Londres, il apprend également la présence de parties de sa famille dans d'autres villes d'Europe.

\footnotetext{
${ }^{26}$ Sandhya préparera et servira un repas pour le groupe thérapeutique, mais elle n’y participera pas, honorant ainsi les règles de commensalité de sa caste.
} 
Karan retrouve ainsi sa position de fils en âge de se marier et en vient à parler de son prochain mariage envisagé par ses parents avec une jeune cousine qui vit dans une capitale européenne. Comme dans un mariage hindou traditionnel, il importe moins de rencontrer la future épouse que de s'assurer de ses références et de la concordance sociale et dharmique des fiancés. Il n'y a paradoxalement aucune contradiction à envisager ce mariage comme un possible élément pour appuyer le dossier administratif de Karan. Ce dernier envisage ainsi son inscription dans un réseau familial décentré élargit de son pays à cette Europe comme territoire d'accueil de la migration tamoule.

\section{La caste et le territoire : la consultation comme port d'amarrage}

Notre réflexion a permis de faire émerger deux termes concernant la traversée traumatique du migrant tamoul du Sri Lanka: la caste et le territoire. A travers deux voix, il s'est s'agit de montrer l'importance de la culture dans l'élaboration psychique de la traversée par les patients. La référence à kalapani vaut moins comme expression intacte d'un tabou traditionnel chez les Hindous de hautes castes que comme métaphore culturelle de la transgression.

La consultation de psycho traumatologie transculturelle se présente comme un dispositif propice pour refaire avec le patient la traversée et, plutôt que de la constituer comme clivage, chaos désorganisateur ou mort symbolique, la repenser comme lien possible. C'est autour de la caste que se condensent les possibilités de lien, et celle-ci s'actualise dans les rapports d'alliance et de commensalité. Le travail clinique procède par inversion du processus de déliaison opéré par le kalapani, et propose une dynamique créative, afin que Sandhya ne vive plus le clivage entre ici et là-bas mais puisse prendre place dans une culture plus globalisée, afin que Karan se sente non plus coupé de sa famille et de sa caste mais compris dans ses réseaux élargis. La reterritorialisation s'actualise alors sur un nouveau plan qui reconfigure les liens et les identités. Ainsi la consultation transculturelle, de groupe ou individuelle, se présente comme espace d'accueil et de holding, port d'amarrage où le patient peut déposer son altérité culturelle en une dynamique d'inscription de sens de cette traversée pour lui permettre l'accostage.

Dans l'odyssée coolie, le poète réunionnais Khal Torabully (1998) rappelle que la métaphore du voyage maudit accompagne toute la vie du travailleur indien qui a quitté sa terre natale pour rejoindre les terres coloniales de labeur ${ }^{27}$. La traversée des eaux noires stigmatise les migrants à la fois comme aventuriers et victimes, et les "castéise » comme coolie. Le concept de la traversée devient ainsi le moment nodal de la migration, l'espace de destruction de l'identité mais aussi de sa régénération, pour une identité moins fixe et plus créative.

\footnotetext{
27 TORABULLY, Khal, "The coolie Odyssey, a voyage in time and space", CARTER, M. \& TORABULLY, K (eds)., Coolitude : an anthology of the Indian Labor Diaspora, Londres, Anthem Press, 1998.
} 


\section{Bibliographie :}

BOKANOWSKI, Thierry, "Traumatisme, traumatique, trauma», Revue française de psychanalyse, $\mathrm{n}^{\circ} 3$, vol. 66, 2002, pp.745-757.

CARTER, Marine; TORABULlY, Khal, Coolitude: an anthology of the Indian Labor Diaspora, Londres: Éd. Anthem Press, 1998, 243p.

DELEUZE, Michel ; GUATTARI, Felix, Capitalisme et schizophrénie, t. 2 : Mille-plateaux, Paris : Éd. Minuit, 1980, 648p.

GOREAU-PONCEAUD, Anthony, "Routes et antiroutes de l'immigration tamoule srilankaise : des camps du Tamil Nadu à la Chapelle », e-migrinter, nº, 2012 , en-ligne.

GOREAU-PONCEAUD, Anthony, «L'immigration sri lankaise en France - Trajectoires, contours et perspectives », Hommes et Migrations, n¹291, 2011, pp.26-39.

IRAGO, Daniel, «Le demandeur d'asile aux prises avec le dehors et le dedans », Cliniques, $\mathrm{n}^{\circ} 2,2011, \mathrm{pp} .139-164$.

KAKAR, Sudhir, The Inner World, A Psycho-Analytic Study of Childhood and Society in India, Delhi: Éd. Oxford Universiy Press, 1982, 280p.

LACHAL, Christian; OUSS-RYNGAERT, Lisa; MORO Marie-Rose, Comprendre et soigner le trauma en situation humanitaire, Paris : Éd. Dunod, 2003, 312p.

MANTOVAN, Giacomo, "Les récits de vie des demandeurs d'asile tamouls. Vers une mémoire collective ? », Hommes et migrations, ${ }^{\circ}$ 1291, 2011, pp.40-50.

MORO, Marie-Rose, Psychothérapie transculturelle des enfants de migrants, Paris: Éd. Dunod, 1998, 214p.

ROUSSEAU, Cécile; SAID, M. Taher; GAGNE, Marie-J ; BIBEAU, Gilles, "Between Myth and Madness, Dreaming the Departure. Premigratory Period for Young Somali Refugee", Culture, Medicine and Psychiatry, n²4, (22), 1998, pp. 385-411.

SAYAD, Abdelmalek, La double absence. Des illusions de l'émigré aux souffrances de l'immigré, Paris : Éd. Seuil, 1999, 437p.

SIRONI, Françoise, Bourreaux et victimes. Psychologie de la torture, Paris : Éd. Odile Jacob, 1999, 280p.

SPENCER, Jonathan, "Anthropology, Politics, and Place in Sri Lanka: South Asian Reflections from an Island Adrift", Samaj, 10/2014. http://samaj.revues.org/3812\#text 